\title{
White matter microstructural abnormalities in euthymic bipolar disorder
}

Karine A. N. Macritchie, Adrian J. Lloyd, Mark E. Bastin, Kamini Vasudev, Peter Gallagher, Rachel Eyre, Ian Marshall, Joanna M. Wardlaw, I. Nicol Ferrier, P. Brian Moore and Allan H. Young

\section{Background}

Abnormal diffusion parameters are reported in specific brain regions and white matter tracts in bipolar disorder.

\section{Aims}

To investigate whether these abnormalities are generalised, and thus evident in large regions of white matter.

\section{Method}

Diffusion parameters were measured at several regions in the corpus callosum and in deep/periventricular white matter in 28 currently euthymic patients with bipolar disorder and controls. White matter hyperintensity loads were assessed.

\section{Results}

Comparing the whole data-sets using the sign test, in the group with bipolar disorder, mean diffusivity was greater at all 15 sites $(P<0.001)$ and fractional anisotropy was reduced at $13(P<0.01)$. The effect of diagnosis was significant for callosal mean diffusivity and fractional anisotropy and for deep/periventricular mean diffusivity (MANCOVA). Comparing individual regions (Mann-Whitney $U$-test), prefrontal and periventricular mean diffusivity were significantly increased; callosal and occipital fractional anisotropy were significantly reduced. Former substance use and lithium were possible confounding factors. Periventricular white matter hyperintensities were associated with significantly increased periventricular mean diffusivity in individuals with bipolar disorder.

\section{Conclusions}

Generalised white matter microstructural abnormalities may exist in bipolar disorder, possibly exacerbated by past substance use and ameliorated by lithium.

\section{Declaration of interest}

None.
Bipolar disorder is associated with brain white matter pathology. Regions of hyperintensity in deep and periventricular white matter are found on magnetic resonance images. ${ }^{1}$ Lateral ventriculomegaly is reported, ${ }^{2}$ apparently unrelated to diminution of the surrounding grey matter structures. ${ }^{3}$ Abnormalities of oligodendrocyte and myelination gene expression ${ }^{4}$ and myelin structure in prefrontal deep (but not gyral) white matter are described. $^{5}$ A systematic review of data from diffusion tensor magnetic resonance imaging (DT-MRI) studies found consistent evidence of white matter microstructural abnormalities in frontal and temporal lobes and tracts in bipolar disorder. ${ }^{6}$

We examined the hypothesis that these abnormalities reflect generalised brain white matter pathology by measuring diffusion parameters using large regions of interest in currently euthymic people with bipolar disorder and in individually matched controls.

\section{Method}

Ethics Committee approval was obtained from the Lothian University Hospitals NHS Trust Local Research Ethics Committee and the Joint Ethics Committee of the Newcastle and North Tyneside Health Authority, the University of Newcastle upon Tyne and the University of Northumbria at Newcastle.

\section{Participants}

Twenty-eight right-handed individuals with bipolar (I or II) disorder were recruited from out-patient clinics and a regional affective disorders service. Twenty-eight right-handed controls, individually matched for age, gender and performance on the National Adult Reading Test (NART) ${ }^{7}$ were recruited.

All participants underwent psychiatric diagnostic interview and medical assessment. All individuals with bipolar disorder had suffered one or more major depressive episodes and at least one episode of hypomania or mania (defined by DSM-IV-TR criteria). ${ }^{8}$ Alcohol and substance use were assessed using the Alcohol Use Disorders Identification Test ${ }^{9}$ and the Substance Misuse Inventory (see Thompson et $a l^{10}$ for description). Contemporaneous smoking status was recorded. Individuals with bipolar disorder were first prospectively verified as euthymic for 4 weeks using the Newcastle Euthymia Protocol (see Thompson et $a l^{10}$ for a description).

Exclusion criteria included dementia, any current comorbid DSM-IV-TR Axis I psychiatric diagnosis, alcohol/substance dependency ever or misuse in the past 6 months, electroconvulsive therapy (ECT) in the previous 6 months, and frank neurological disorders. No changes in psychotropic medication were permitted within 1 month of the euthymia protocol. Control participants were excluded if they or any first-degree relative had an Axis I psychiatric disorder.

\section{Neuroimaging}

Magnetic resonance imaging data were obtained using a GE Signa LX 1.5 T (General Electric, Milwaukee, WI, USA) clinical scanner with a self-shielding gradient set $(22 \mathrm{mT} / \mathrm{m}$ maximum gradient strength) and manufacturer-supplied 'birdcage' quadrature head coil. Standard structural imaging and a DT-MRI protocol based on spin-echo echo-planar (EP) imaging was performed. For all sequences the field of view was $24 \times 24 \mathrm{~cm}$ with slices positioned contiguously in the axial plane.

The structural imaging consisted of two fast spin-echo (FSE) sequences, a dual echo sequence that gave proton density and $T_{2}$-weighted images (TR $5000 \mathrm{~ms}, \mathrm{TE} 15$ and $102 \mathrm{~ms}$, slice thickness $5 \mathrm{~mm}$ and acquisition matrix $256 \times 256$ ), and a $T_{1^{-}}$ weighted sequence (TR $650 \mathrm{~ms}$, TE $9 \mathrm{~ms}$, slice thickness $5 \mathrm{~mm}$ and acquisition matrix $256 \times 256)$. In the DT-MRI protocol, sets of contiguous axial EP images $\left(b=0\right.$ and $\left.1000 \mathrm{~s} / \mathrm{mm}^{2}\right)$ were 
collected with diffusion gradients applied sequentially along six non-collinear directions. Five acquisitions consisting of a baseline $T_{2}$-weighted EP image and 6 diffusion-weighted EP images, a total of $35 \mathrm{EP}$ images, were collected per slice position. The acquisition parameters for the EP imaging sequence were 51 axial slices of $3 \mathrm{~mm}$ thickness, an acquisition matrix of $96 \times 96$ (zero filled to $256 \times 256$ to give a voxel dimension of $0.94 \times 0.94 \times 3 \mathrm{~mm}$ ), a TR of $7.5 \mathrm{~s}$ and a TE of $93.6 \mathrm{~ms}$.

\section{Region of interest analysis}

From the DT-MRI data, the apparent diffusion tensor of water (D) was calculated in each voxel from the signal intensities in the component EP images with bulk patient motion and eddy current induced artifacts removed by registering the diffusion weighted to the first $T_{2}$-weighted EP volume for each participant using FLIRT (FMRIB, Oxford, UK; www.fmrib.ox.ac.uk). Maps of mean diffusivity and fractional anisotropy were generated on a voxel-by-voxel basis from the sorted eigenvalues of $\mathrm{D}$ and converted into Analyze format.

Using Analyze AVW.4.0 for Windows (http://Analyzedirect. com, Rochester, MN, USA), object maps were constructed on contiguous axial slices of the structural $T_{2}$-weighted EP images by observers masked to diagnosis and DT-MRI data. Regions of interest (ROIs) were defined in two object maps: the callosal and the deep/ periventricular white matter object maps. Each object map was constructed by the same observers in all participants.

For the callosal object map (Fig. 1), ROIs were prescribed bilaterally in the genu ('anterior callosum'), the splenium ('posterior callosum') and the body, commencing from the most inferior axial slice on which the corpus callosum was seen to cross the midline and moving superiorly through the slices until the callosum crossed the midline as a single central structure. From this point superiorly, the regions were combined into one ROI, termed 'the callosal body'. In the final analysis, all callosal regions were also combined to form a 'total' corpus callosum ROI.

A deep/periventricular object map comprised five ROIs, prescribed bilaterally in standardised positions in each slice (Fig. 2): prefrontal deep white matter regions, two periventricular positions (adjacent to the anterior horn of the lateral ventricles), a central region in the area of the centrum semiovale and an occipital region. The ROIs for the prefrontal, central and occipital regions were, where possible, obtained from the same axial slices, commencing inferiorly at the level of the subgenual anterior cingulate cortex. Where this was not possible, the closest available slices were used. The ROIs extended superiorly through contiguous slices to the last slice in which white matter was visible with no encroaching grey matter from the overlying cortex. The periventricular ROIs extended from the most inferior but one slice on which the anterior horns of the lateral ventricles could be seen to the most superior slice in which the horns remained clearly defined.

Object maps were transferred to the spatially co-registered diffusion maps (Figs 1 and 2). Mean diffusivity and fractional anisotropy for each ROI were calculated using the Analyze programme.

\section{Assessment of white matter abnormalities}

Deep white matter and periventricular hyperintensity loads were assessed by three masked observers using Fazekas' scale ${ }^{11}$ on FSE

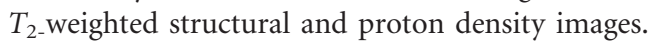

$T_{2}$ image

(a)

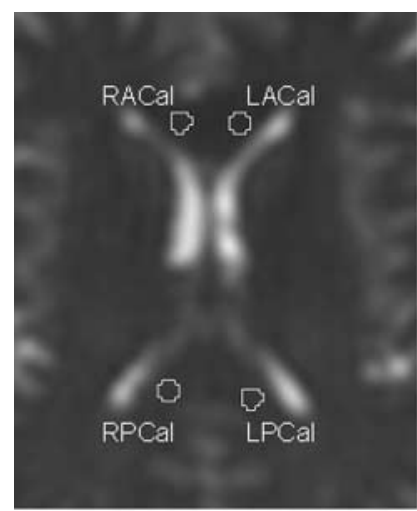

(b)

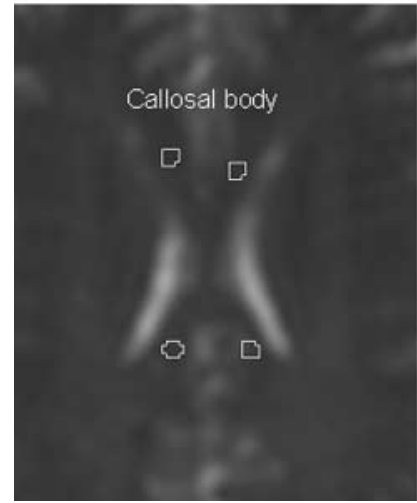

Mean diffusivity map
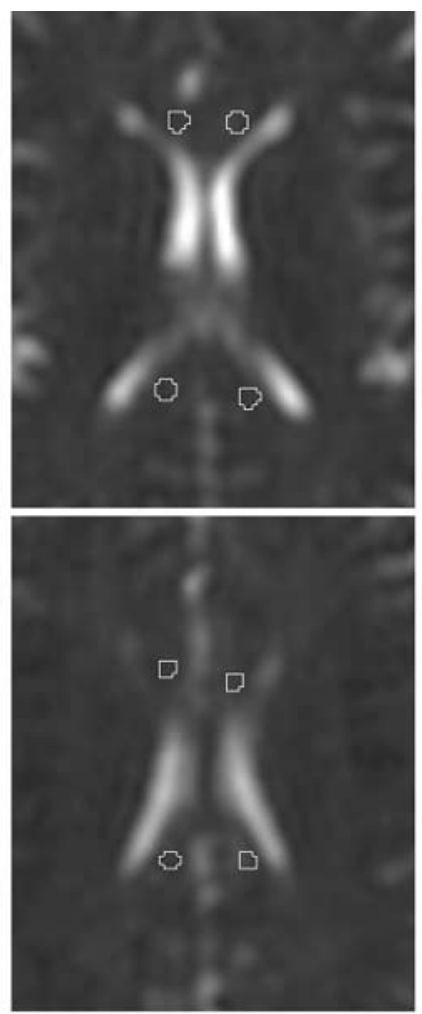

Fractional anisotropy map
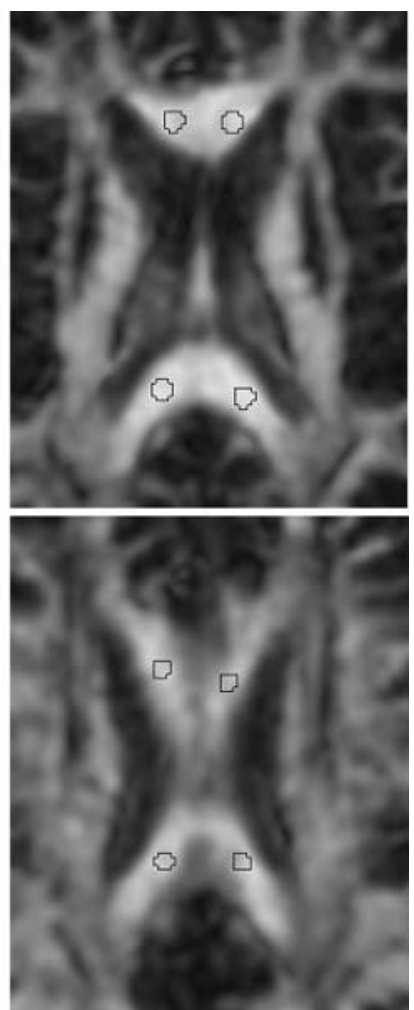

Fig. 1 The callosal object map: transposition to diffusion parameter maps.

(a) Level of the mid-lateral ventricle and the callosal splenium and genu; (b) level of the upper lateral ventricle and the callosal body. LACal, left anterior callosum; LPCal, left posterio callosum; RACal, right anterior callosum; RPCal, right posterior callosum. 


\section{Data analysis}

Data were analysed using SPSS for Windows version 16 (2007). For comparisons between two groups, independent $t$-tests were applied for normally distributed data. Non-parametric tests were used when data were not normally distributed and for DT-MRI data. To compare diffusion parameter data-sets as a whole, the sign test and MANCOVAs were used. For the MANCOVAs, four models were used: these were fractional anisotropy and mean diffusivity data from the independently measured callosal regions (the anterior and posterior regions and the body) and from the deep/periventricular white matter regions (the prefrontal, periventricular, central and occipital regions). For comparison of individual regions, the Mann-Whitney $U$-test was applied. Categorical data were compared between groups using the chisquared test or Fisher's exact test. For all comparisons, a significance value of $P<0.05$ was used.

\section{Results}

\section{Participants}

Twenty patients had bipolar I disorder; eight had bipolar II disorder. The mean (s.d.) age at illness onset was 24.2 (7.0) years and illness duration was 18.3 (10.7) years. Individuals had been euthymic for 15.0 (26.8) months (range 1.5 months to 12 years). Their mean number of episodes was 13.6 (9.7) (manic 3.3 (3.5); hypomanic 2.4 (4.4); depressive 7.8 (8.1)).
Each group comprised 16 men and 12 women. The groups did not differ significantly in age (mean (s.d.): bipolar group 43.0 (11.5) years, controls 43.0 (11.7) years) or in the NART estimate of premorbid IQ (bipolar group 113 (10.0), controls 113 (7.8)).

Complete data on alcohol and substance use were available from 54 of the 56 participants. None had a history of alcohol or substance dependency/misuse in the past 6 months. Significantly more people with bipolar disorder had a past history of any illicit substance use: 14 of 28 compared with 4 of 27 (Fisher's exact test $P<0.01$ ). A significantly higher proportion of participants in the bipolar group had a prior history of alcohol misuse: 7 of 27 compared with 1 of 27 controls (Fisher's exact test $P=0.05$ ). Of these, one patient with bipolar disorder and his control did have a history of alcohol dependency: both had been abstinent for more than 20 years. The proportion of smokers did not differ: 7 of 26 in the bipolar group compared with 4 of 27 controls (Fisher's exact test $P=0.53$ ).

\section{Treatment}

None of the patients with bipolar disorder was psychotropic naive. Two were medication-free. Five received monotherapy; 21 were on combination treatment. Sixteen patients took lithium. Ten individuals not on lithium received other psychotropic medication: of these, nine were on anticonvulsants. Six patients were on antipsychotic medication. Ten had received ECT in the past.
$T_{2}$ image

(a)

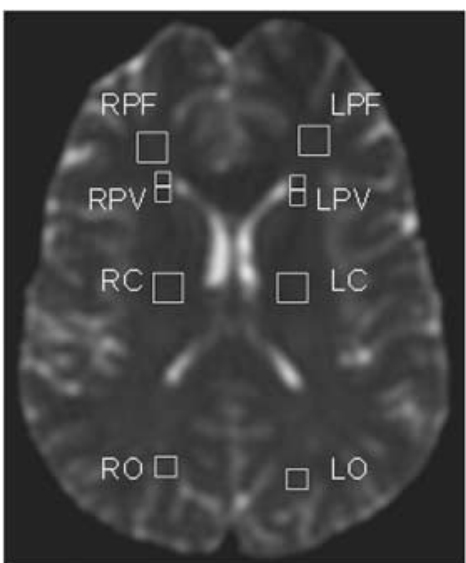

(b)

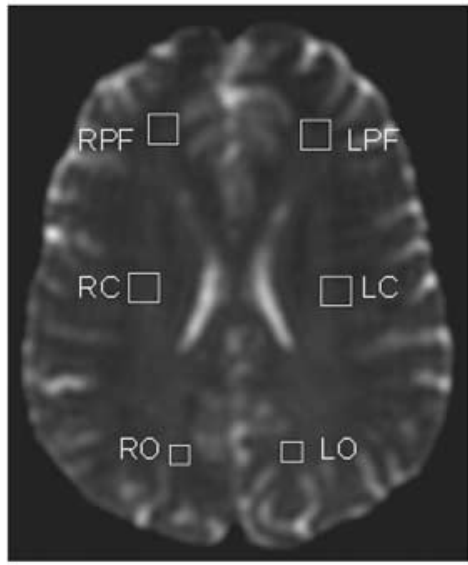

Mean diffusivity map
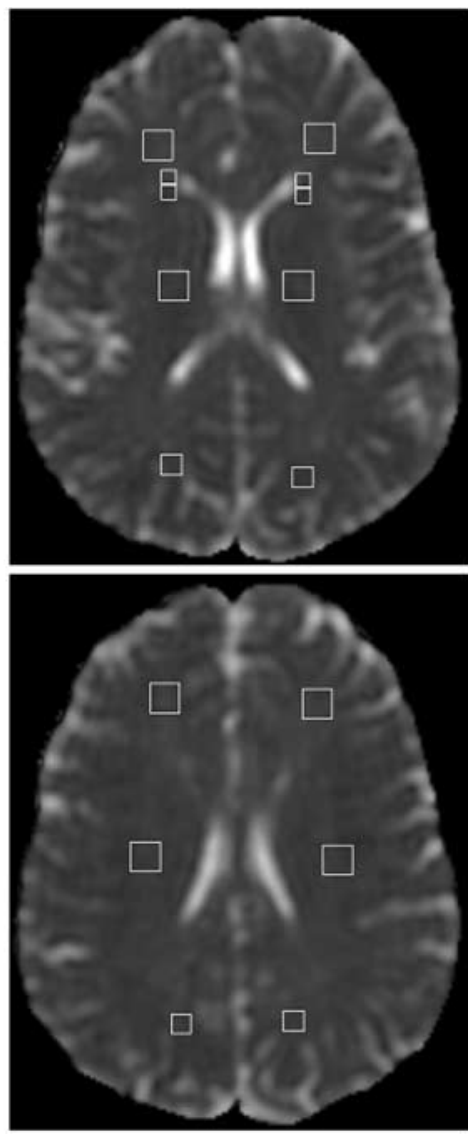

Fractional anisotropy map
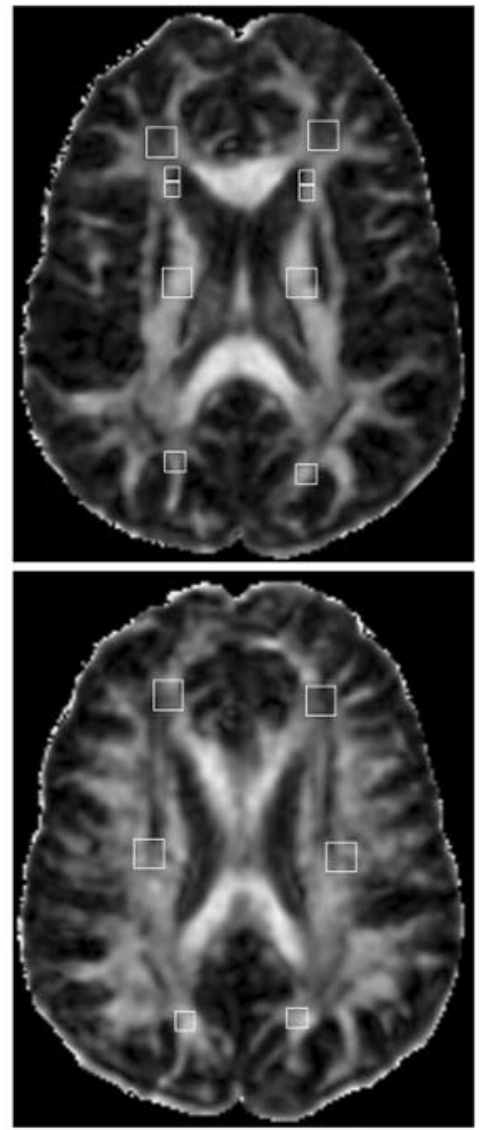

Fig. 2 Object map of the deep and periventricular white matter: transposition to diffusion parameter maps.

(a) Level of the mid-lateral ventricles, the callosal splenium and genu; (b) level of the upper lateral ventricles and the callosal body. LPF, left prefrontal; RPF, right prefrontal; LPV, left periventricular (1) and (2); RPV, right periventricular (1) and (2); LC, left central; RC, right central; LO, left occipital; RO, right occipital. In each case, the periventricular region 1 was sited anterior to the periventricular region 2. 


\section{DT-MRI data: whole-group comparisons}

Intrarater test-retest reliability testing showed mean percentage differences ranging from 0.6 to $7.7 \%$ for mean diffusivity and 1.2 to $8.6 \%$ for fractional anisotropy.

Comparing the DT-MRI data-sets as a whole (Table 1), the number of sites at which mean diffusivity in the bipolar group exceeded that of controls (all 15) was highly significant using the sign test $(P<0.001$; two-tailed). Similarly, fractional anisotropy in controls exceeded that in the bipolar group at 13 of the 15 sites (sign test: $P=0.008$; two-tailed) (Table 1 ). Using MANCOVAs, the effect of diagnosis was significant for callosal mean diffusivity (Pillai's trace $0.231, F=2.94$, d.f. $=5,49, P=0.02$ ) and fractional anisotropy (Pillai's trace $0.305, F=4.31$, d.f. $=5,49, P=0.003$ ) and for the deep/periventricular white matter mean diffusivity (Pillai's trace $0.32, F=2.06$, d.f. $=10,44, P=0.05$ ) in which age was also a significant covariate. The effect of diagnosis was not significant for deep/periventricular white matter fractional anisotropy (Pillai's trace $0.25, F=1.48$, d.f. $=10,44, P=0.18$ ).

Comparing individual ROIs, mean diffusivity was significantly increased in three callosal regions, in the total corpus callosum and bilaterally in the prefrontal and in the periventricular regions in the bipolar group. Fractional anisotropy was significantly reduced in all the regions of the corpus callosum and in the left occipital region.
The pattern of results was similar for the bipolar I subgroup analysis using the sign test $(P<0.01$ for mean diffusivity and fractional anisotropy) and for the individual ROI comparisons although fewer callosal mean diffusivity comparisons were significantly different (online Table DS1).

\section{DT-MRI data: alcohol and illicit substance subgroup comparisons}

Mean diffusivity was significantly increased in the right prefrontal region and in two periventricular regions in patients with bipolar disorder and a positive history of any illicit substance use $(n=14)$ when compared with patients with no such history $(n=14)$. Fractional anisotropy did not differ significantly (online Table DS2).

We compared the 14 people with bipolar disorder with no history of illicit substance use with their matched controls. Using the sign test, mean diffusivity was greater at 13 sites and fractional anisotropy was reduced at 14 sites $(P<0.01$; two-tailed in each case). Comparing individual ROIs ( $U$-test), two callosal regions and the combined ROI had significantly lower fractional anisotropy. Prefrontal fractional anisotropy decreases also approached significance. However, no prefrontal and periventricular pattern

Table 1 Mean diffusivity and fractional anisotropy for the bipolar group and their controls individually matched for age, gender and performance on National Adult Reading Test: main-group comparison

\begin{tabular}{|c|c|c|c|}
\hline & \multicolumn{2}{|c|}{ Mean (s.d.) } & \multirow{2}{*}{$\begin{array}{l}\text { 2-tailed } P \text { (U-test) } \\
\text { Regions of interest }\end{array}$} \\
\hline & Bipolar group, $n=28$ & Control group, $n=28$ & \\
\hline \multicolumn{4}{|l|}{ Mean diffusivity $\times 10^{-6} \mathrm{~mm}^{2} / \mathrm{s}$} \\
\hline Corpus callosum (total) & $875(51)$ & $846(40)$ & $0.04^{*}$ \\
\hline Corpus callosum (body) ${ }^{a}$ & $988(132)$ & $953(87)$ & 0.39 \\
\hline Left anterior $^{\mathrm{a}}$ & $854(58)$ & $839(57)$ & 0.22 \\
\hline Right anterior $^{a}$ & $864(62)$ & $834(52)$ & $0.04^{*}$ \\
\hline Left posterior ${ }^{a}$ & $829(63)$ & $805(73)$ & $0.046^{*}$ \\
\hline Right posterior ${ }^{a}$ & $840(90)$ & $785(71)$ & $0.01^{*}$ \\
\hline Left prefrontal $^{\mathrm{b}}$ & $776(30)$ & $758(26)$ & $0.01 *$ \\
\hline Right prefrontal ${ }^{\mathrm{b}}$ & 767 (29) & 747 (29) & $0.004^{*}$ \\
\hline Left periventricular $(1)^{b}$ & $909(51)$ & $878(45)$ & $0.02 *$ \\
\hline Left periventricular $(2)^{\mathrm{b}}$ & $875(88)$ & $800(47)$ & $0.001 *$ \\
\hline Right periventricular (1) ${ }^{\mathrm{b}}$ & $853(43)$ & $828(47)$ & $0.046^{*}$ \\
\hline Right periventricular $(2)^{b}$ & $788(42)$ & $748(45)$ & $0.001 *$ \\
\hline Left central $^{\mathrm{b}}$ & $717(20)$ & 708 (19) & 0.15 \\
\hline Right central ${ }^{\mathrm{b}}$ & 707 (17) & $702(20)$ & 0.26 \\
\hline Left occipital ${ }^{\mathrm{b}}$ & $732(25)$ & $720(29)$ & 0.053 \\
\hline Right occipital ${ }^{\mathrm{b}}$ & $738(26)$ & 729 (25) & 0.23 \\
\hline \multicolumn{4}{|l|}{ Fractional anisotropy $\times 10^{-4}$} \\
\hline Corpus callosum (total) & 6727 (444) & 7206 (404) & $<0.001 *$ \\
\hline Corpus callosum (body) ${ }^{c}$ & $5921(614)$ & 6475 (569) & $0.003^{*}$ \\
\hline Left anterior ${ }^{\mathrm{C}}$ & $6918(483)$ & 7206 (499) & $0.04^{*}$ \\
\hline Right anterior ${ }^{\mathrm{C}}$ & 6718 (537) & 7201 (473) & $0.002^{*}$ \\
\hline Left posterior ${ }^{c}$ & $7215(716)$ & 7689 (623) & $0.007^{*}$ \\
\hline Right posterior ${ }^{\mathrm{C}}$ & $6954(863)$ & 7598 (555) & $0.001 *$ \\
\hline Left prefrontal $^{d}$ & 3096 (242) & 3210 (295) & 0.08 \\
\hline Right prefrontal ${ }^{\mathrm{d}}$ & $3143(251)$ & 3219 (233) & 0.26 \\
\hline Left periventricular (1) ${ }^{\mathrm{d}}$ & 3092 (628) & 3214 (595) & 0.60 \\
\hline Left periventricular $(2)^{\mathrm{d}}$ & 3509 (630) & 3616 (698) & 0.67 \\
\hline Right periventricular (1) ${ }^{\mathrm{d}}$ & 3199 (633) & 3171 (701) & 0.72 \\
\hline Right periventricular (2) ${ }^{\mathrm{d}}$ & 3453 (848) & 3412 (696) & 0.58 \\
\hline Left central ${ }^{d}$ & 4302 (394) & $4433(441)$ & 0.27 \\
\hline Right central $^{\mathrm{d}}$ & $4313(388)$ & 4438 (424) & 0.53 \\
\hline Left occipital ${ }^{d}$ & $3858(383)$ & 4042 (398) & $0.05^{\star}$ \\
\hline Right occipital ${ }^{d}$ & $3892(360)$ & 3992 (396) & 0.39 \\
\hline
\end{tabular}


of significantly increased mean diffusivity was evident (online Table DS2).

When individuals with a history of alcohol dependency or misuse were excluded from the main-group analysis, the results were broadly similar to that of the main-group comparisons.

\section{DT-MRI Data: lithium subgroup comparisons}

Comparing data-sets from the lithium- and non-lithium-treated bipolar subgroups directly (online Table DS3), mean diffusivity in the non-lithium treated group was higher than in the lithium group at 12 of the 15 sites (sign test: $P=0.04$; two-tailed). Fractional anisotropy was lower at 10 of the 15 sites (sign test: $P=0.30$; two-tailed). Comparing individual ROIs directly, no significant differences were found in diffusion parameters.

In the non-lithium-treated bipolar group $(n=12)$ compared with their controls (online Table DS3), the pattern of increased prefrontal, periventricular and callosal mean diffusivity previously observed in the main-group comparison persisted. This pattern was not seen in the lithium-treated group $(n=16)$ compared with their control group. For fractional anisotropy, the pattern of results did not differ markedly between treatment groups, although more callosal differences retained statistical significance in the larger lithium-treated group. There was no difference in the proportions of individuals with a history of substance use in these subgroups ( 8 of 16 individuals on lithium compared with 6 of 12 individuals not on lithium).

\section{White matter hyperintensities}

Twelve of the 28 patients with bipolar disorder had deep white matter hyperintensities compared with thirteen of the control group. Eleven of the 28 patients with bipolar disorder had abnormal periventricular white matter hyperintensities compared with seven of the control group. There was no significant difference in comparing these proportions $\left(\chi^{2}=0.07\right.$ for deep white matter, $P=0.79 ; \chi^{2}=1.31$ for periventricular hyperintensities, $P=0.25$ ). Periventricular mean diffusivity was significantly increased at two sites in people in the bipolar group with white matter hyperintensities compared with those without (right periventricular region 1: mean (s.d.) $879.93(55.8) \times 10^{-6} \mathrm{~mm}^{2} / \mathrm{s}$ v. $836.48(22.7) \times 10^{-6} \mathrm{~mm}^{2} / \mathrm{s}$ respectively, $P=0.03$; the left periventricular region 2: $919.0(81.0) \times 10^{-6} \mathrm{~mm}^{2} / \mathrm{s} \quad$ v. 847.34 $\left.(82.1) \times 10^{-6} \mathrm{~mm}^{2} / \mathrm{s}, P=0.03\right)$.

\section{Discussion}

Examining the data-sets as a whole, mean diffusivity was increased at all independently analysed sites in the main bipolar group; fractional anisotropy was lower at 13 of the 15 sites. Both these proportions are highly significant using the sign test. On MANCOVA, callosal fractional anisotropy and mean diffusivity and deep/periventricular mean diffusivity showed significant differences between the groups. Comparing individual ROIs, in the bipolar group, mean diffusivity was significantly increased bilaterally in the prefrontal and periventricular regions, the genu and the splenium, and in a combined total callosal region of interest. Fractional anisotropy was significantly decreased in all callosal regions and in the dominant occipital region. The proportions of individuals with white matter hyperintensities did not differ between the groups. There was evidence that a history of past substance use and lithium may be confounding factors but they do not explain all the changes observed in the main-group analysis.

\section{Methodological issues}

This work has several methodological strengths. Participants with bipolar disorder were prospectively verified as euthymic. Through individual matching of participants in the bipolar and control groups, main and most subgroup analyses were controlled for the potentially confounding factors of age, handedness, gender and premorbid IQ. However, several limitations remain. The duration and severity of the course of bipolar illness varied: nonetheless, all were euthymic at scanning. Psychotropic regimens differed: most people on lithium were on other medications, and most non-lithium-treated individuals received other psychotropic agents. Participants in the bipolar group had a greater frequency of past alcohol and previous illicit substance use than the controls.

Two other methodological issues are worth mentioning. First, in comparison to the callosal ROIs and ROIs used in previous diffusion tensor imaging studies, the prefrontal, central and occipital ROIs were large and may have exhibited greater heterogeneity in fibre orientation. Consequently, this study may be underpowered to demonstrate differences in fractional anisotropy in some regions.

Second, given the number of statistical comparisons, it might be argued that a Bonferroni correction for multiple comparisons should be applied. However, its application may be inappropriate when variables are not independent: ${ }^{12}$ such correction risks that a pattern of structural abnormality is discounted through a Type II error.

\section{Are these data evidence of a generalised white matter abnormality in bipolar disorder?}

Examination of the diffusion data-sets as a whole using the sign test and the MANCOVA supports the hypothesis that a generalised abnormality of white matter structure exists in bipolar disorder. White matter comprises an oligodendrocyte network supporting neuronal axons, a substantial proportion of which are myelinated. Studies of spinal cord white matter in myelin deficient rats have shown that myelination is an important influence on mean diffusivity and fractional anisotropy. ${ }^{13}$ Fractional anisotropy is correlated with axon number and density in peripheral nerves. ${ }^{14}$ Elevated mean diffusivity is assumed to reflect increased extracellular fluid. To our knowledge, the effects of the extensive oligodendrocyte network itself on water molecule diffusion have not been investigated, but they are potentially substantial: one oligodendrocyte may possess projections myelinating up to 50 axons. ${ }^{15}$

The diffusion data in the bipolar group may reflect fewer or thinner axons or abnormalities affecting oligodendrocytes and myelination. Spectroscopy studies, structural and functional MRI ${ }^{16}$ histopathological studies ${ }^{5,17,18}$ and an oligodendrocyte and myelination gene expression study ${ }^{4}$ support these possibilities. We suggest that changes to the water homeostasis system, particularly in the periventricular region, ${ }^{19}$ and/or structural diminution of the extensive oligodendrocyte network may also contribute to changes in mean diffusivity in bipolar disorder.

\section{Specific regional group comparisons}

For individual ROI comparisons, the prefrontal and periventricular distribution of the significant regional differences in mean diffusivity is similar to those found in a recent voxel-based DT-MRI study. ${ }^{20}$ Fractional anisotropy was decreased in all callosal regions, consistent with most, but not all, previous studies. ${ }^{6}$

Our study raises questions regarding the relationship between periventricular mean diffusivity and periventricular hyperintensities in bipolar disorder. Hyperintensities are likely to be macrostructural signs of pathological processes affecting white matter. We know of 
no published histological studies of periventricular white matter hyperintensities in bipolar disorder, but work undertaken in individuals with late-life depression showed three types of pathology: differing degrees of myelination, cerebral ischaemia with associated demyelination and ependymal loss. No histological features differentiated individuals with depression from controls. ${ }^{21}$ We found no difference in the proportions of participants with periventricular hyperintensities between the groups. However, periventricular mean diffusivity was significantly increased in the main bipolar group compared with controls. Subtle white matter abnormalities may exist in bipolar disorder not evident in hyperintensities on conventional MRI scans. Within the bipolar group, periventricular white matter hyperintensities were associated with significantly increased mean diffusivity. This is consistent with the report of increased mean diffusivity and decreased fractional anisotropy in hyperintensities found in individuals with depression and controls. ${ }^{22}$

\section{The illicit substance use post hoc analyses}

Half of the bipolar group had a lifetime history of illicit substance use. The rate of past substance use was higher than the $41 \%$ rate of lifetime misuse reported in people with bipolar I disorder in the Epidemiologic Catchment Area Study, ${ }^{23}$ possibly because we counted even brief experimental exposure. In our study, no participants had active substance misuse or dependency or any alcohol/substance dependency ever (with the two exceptions described above); alcohol and/or substance misuse in the previous 6 months were exclusion criteria. These subgroup analyses suggest that a history of substance use may be a confounding factor: the extent to which it influenced the results of these analyses is unclear. Decreased frontal white matter volume and preliminary evidence of frontotemporal diffusion abnormalities have been reported in association with severe chronic and ongoing substance misuse, ${ }^{24,25}$ but the effects of past use remain to be determined.

\section{The lithium subgroup post hoc analyses}

There are several possible reasons for the findings of the lithium subgroup analysis. One methodological concern is that they may arise through the magnetic resonance effects of lithium. Previous work suggests that lithium may affect quantitative MRI parameters. Elevated spin-lattice relaxation time $\left(T_{1}\right)$ values were reported in the frontal and temporal lobes of people with bipolar disorder compared with controls, a difference which disappeared following lithium therapy. ${ }^{26}$ Two subsequent studies showed no significant difference in $T_{1}$ in the white matter of people with bipolar disorder predominantly on lithium therapy compared with controls, ${ }^{27,28}$ but frontal white matter $T_{1}$ was increased in unipolar depressed people not on lithium. ${ }^{27}$ The findings of these studies and ours may reflect lithium magnetic resonance effects: however, $T_{1}$ does not appear to be directly correlated with mean diffusivity in normal-appearing white matter. ${ }^{29}$

Another possible explanation for our observation is that white matter microstructural abnormalities may be associated with lithium non-responsiveness and that the non-lithium-treated group were inadvertently 'pre-selected' to demonstrate diffusion parameter differences. Indices of this clinical factor were not recorded in this study, but should be examined in future work.

Finally, our data may reflect a biological effect of lithium. Little information exists on the effects of lithium on water homeostatic mechanisms, but one study reported an increase in brain water in lithium-treated rats ${ }^{30}$ that is not consistent with our findings. Alternatively, our data may be evidence of the trophic action of lithium on neuronal cells ${ }^{31}$ or possibly a trophic effect on the deep white matter oligodendrocyte network.
Histopathological evidence of medication effects on glial cell structure and function is scant. In one small study, ${ }^{32}$ two patients with bipolar disorder receiving neither lithium nor valproate exhibited a significant and large reduction in amygdalar glial/neuron ratio and glial density in contrast with the main bipolar group.

\section{Future research}

Do white matter microstructural abnormalities represent vulnerability factors, traits or scars of bipolar disorder? Abnormal diffusion parameters in children with bipolar disorder, in those at high risk of the illness ${ }^{33}$ and in medication-free adolescents experiencing their first episode of mania ${ }^{34}$ suggest that some abnormalities are present early and before brain myelination is complete.

Promising avenues for future research include the roles of oligodendrocyte gene expression and oligodendrocyte dysfunction, ${ }^{4}$ glutamate neuronal and oligodendrocyte toxicity ${ }^{35,36}$ and glucocorticoid activity. ${ }^{37}$ Genetic control of white matter integrity in bipolar disorder has been highlighted recently. ${ }^{38}$ The effects of lithium and other agents, including illicit substances, on oligodendrocyte structure and function and brain water homeostasis should be investigated further. Longitudinal volumetric and DT-MRI studies of individuals with well-characterised bipolar disorder after their first episode of mania are required. Further histopathological studies of the deep white matter in people with well-characterised bipolar disorder may determine the nature of these putative microstructural abnormalities.

\begin{abstract}
Karine A. N. Macritchie, BSC(Hons), MB ChB, MD, MRCPsych, Adrian J. Lloyd, MB BS, MRCPsych, MD(Hons), Psychobiology Research Group, Institute of Neuroscience, Newcastle University; Mark E. Bastin, DPhil, Medical and Radiological Sciences and SFC Brain Imaging Research Centre, University of Edinburgh and Scottish Imaging Network, A Platform for Scientific Excellence (SINAPSE) Collaboration; Kamini Vasudev, MD, DNB(Pharmacology), MRCPsych, Peter Gallagher, BSC(Hons), MPhil, Rachel Eyre, BSc, MRes, Psychobiology Research Group, Institute of Neuroscience, Newcastle University, UK; Ian Marshall, PhD, CEng, CSci, FIPEM, Medical and Radiological Sciences and SFC Brain Imaging Research Centre, University of Edinburgh and Scottish Imaging Network, A Platform for Scientific Excellence (SINAPSE) Collaboration; Joanna M. Wardlaw, BSC, MB ChB, FRCR, FRCP, FMedSci, MD, Division of Clinical Neurosciences and SFC Brain Imaging Research Centre, $\mathrm{MD}$, Division of Clinical Neurosciences and SFC Brain Imaging Research Centre,
University of Edinburgh and Scottish Imaging Network, A Platform for Scientific Excellence (SINAPSE) Collaboration; I. Nicol Ferrier, BSC(Hons), MD(Hons), FRCP(Ed), FRCPsych, P. Brian Moore, MB BS, BSC, PhD, FRCPsych, Allan H. Young, MB ChB, MPhil, PhD, FRCPsych, FRCPS, Psychobiology Research Group, Institute of
\end{abstract} Neuroscience, Newcastle University, UK

Correspondence: Karine A.N. Macritchie, Institute of Mental Health Department of Psychiatry, University of British Columbia, Suite 430

Strangway Building, 5950 University Boulevard, Vancouver, BC V6T 1Z3, Canada. Email: karine.macritchie@gmail.com

First received 18 Aug 2008, final revision 12 Sep 2009, accepted 26 Sep 2009

\section{Funding}

This work was funded by a Stanley Medical Research Institute Grant. All MRI data were collected at the SFC Brain Imaging Research Centre, University of Edinburgh (www.sbirc.ed.ac.uk), part of the SINAPSE Collaboration (Scottish Imaging Network: A Platform for Scientific Excellence; http://www.sinapse.ac.uk) funded by the Scottish funding council and Chief Scientist Office and which also part funds Professor JM Wardlaw.

\section{Acknowledgements}

The neuroimaging protocol was administered by Mrs A. Blane and Mrs E. Cowie and their team

\section{References}

1 Monkul ES, Malhi GS, Soares JC. Anatomical MRI abnormalities in bipola disorder: do they exist and do they progress? Aust N Z J Psychiatry 2005; 39 : 222-6. 
2 Arnone D, Cavanagh J, Gerber D, Lawrie SM, Ebmeier KP, McIntosh AM. Magnetic resonance imaging studies in bipolar disorder and schizophrenia: meta-analysis. Br J Psychiatry 2009; 195: 194-201.

3 Strakowski SM, DelBello MP, Zimmerman ME, Getz GE, Mills NP, Ret J, et al. Ventricular and periventricular structural volumes in first- vs multiple-episode bipolar disorder. Am J Psychiatry 2002; 159: 1841-7.

4 Tkachev D, Mimmack ML, Ryan MM, Wayland M, Freeman T, Jones PB, et al. Oligodendrocyte dysfunction in schizophrenia and bipolar disorder. Lancet 2003; 362: 798-805.

5 Regenold WT, Phatak P, Marano CM, Gearhart L, Viens CH, Hisley KC. Myelin staining of deep white matter in the dorsolateral prefrontal cortex in schizophrenia, bipolar disorder and unipolar major depression. Psychiatry Res 2007; 151: 179-88.

6 Sexton CE, MacKay CE, Ebmeier K.P. A systematic review of diffusion tensor imaging studies in affective disorders. Biol Psychiatry 2009; 66: 814-23.

7 Nelson HE. National Adult Reading Test, NART. nferNelson, 1982.

8 American Psychiatric Association. Diagnostic and Statistical Manual of Mental Disorders (4th edn, revised) (DSM-IV-TR). APA, 2000.

9 Reinert DF, Allen JP. The Alcohol Use Disorders Identification Test (AUDIT): a review of recent research. Alcohol Clin Exp Res 2002; 26: 272-9.

10 Thompson JM, Gallagher P, Hughes JH, Watson S, Gray JM, Ferrier IN, et al. Neurocognitive impairment in euthymic patients with bipolar affective disorder. Br J Psychiatry 2005; 186: 32-40.

11 Fazekas F, Chawluk JB, Alavi A, Hurtig HI, Zimmerman RA. MR signal abnormalities at $1.5 \mathrm{~T}$ in Alzheimer's dementia and normal aging. Am J Neuroradiol 1987; 8: 421-6.

12 Perneger TV. What's wrong with Bonferonni adjustments. BMJ 1998; 316 $1236-8$

13 Gulani V, Webb AG, Duncan ID, Lauterbur PC. Apparent diffusion tensor measurements in myelin-deficient rat spinal cords. Magn Reson Med 2001; 45: 191-5.

14 Takagi T, Nakamura M, Yamada M, Hikishima K, Momoshima S, Fujiyoshi K, et al. Visualization of peripheral nerve degeneration and regeneration: monitoring with diffusion tensor tractography. Neuroimage 2009; 44: 884-92.

15 Burkitt HG, Young B, Heath JW. Central nervous system. In Wheater's Functional Histology: A Text and Colour Atlas (eds HS Burkitt, B Young JW Heath). Churchill Livingstone, 1993.

16 Strakowski SM, Delbello MP, Adler CM. The functional neuroanatomy of bipolar disorder: a review of neuroimaging findings. Mol Psychiatry 2005; 10 105-16.

17 Harrison PJ. The neuropathology of primary mood disorder. Brain 2002; 125 1428-49.

18 Uranova NA, Vostrikov BM, Orlovskaya DD, Rachmanova VI. Oligodendroglia density in the prefrontal cortex in schizophrenia and mood disorders: a study from the Stanley Neuropathology Consortium. Schizophr Res 2004; 67: 269-75.

19 Tait MJ, Saadoun S, Bell BA, Papadopoulos MC. Water movements in the brain: role of aquaporins. Trends Neurosci 2008; 31: 37-43.

20 Bruno S, Cercignani M, Ron MA. White matter abnormalities in bipolar disorder: a voxel-based diffusion tensor imaging study. Bipolar Disord 2008; 10: $460-8$.
21 Thomas AJ, O'Brien JT, Barber R, McMeekin W, Perry R. A neuropathological study of periventricular white matter hyperintensities in major depression. J Affect Disord 2003; 76: 49-54.

22 Taylor WD, Payne ME, Krishnan RR, Wagner HR, Provenzale JM, Steffens DC, et al. Evidence of white matter tract disruption in MRI hyperintensities. Biol Psychiatry 2001; 50: 179-83.

23 Regier DA, Farmer ME, Rae DS, Licke BZ, Keith SJ, Judd LL, et al. Comorbidity of mental disorders with alcohol and other drug abuse. Results from the Epidemiologic Catchment Area (ECA) study. JAMA 1990; 264: 2511-8.

24 Schlaepfer TE, Lancaster E, Heidbreder R, Strain EC, Kosel M, Fisch H-U, et al. Decreased frontal white matter volume in chronic substance misuse. Int J Neuropsychopharmacol 2006; 9: 147-53.

25 Ashtari M, Cervellione $\mathrm{K}$, Cottone J, Ardekain BAA, Kumra S. Diffusion abnormalities in adolescents with a history of heavy cannabis use. J Psychiatric Res 2009; 43: 189-204.

26 Rangel-Guerra RA, Perez-Payan H, Minkoff L, Todd LE. Nuclear magnetic resonance in bipolar affective disorders. Am J Neuroradiol 1983; 4: 229-31.

27 Dolan RJ, Poynton AM, Bridges PK, Trimble MR. Altered magnetic resonance white-matter $\mathrm{T}_{1}$ values in patients with affective disorder. $\mathrm{Br} J$ Psychiatry 1990; 157: 107-10.

28 Besson JA, Henderson JG, Foreman El, Smith FW. An NMR study of lithium responding manic depressive patients. Magn Reson Imaging 1987; 5: 273-7.

29 Bastin ME, Sinha S, Whittle IR, Wardlaw JM. Measurements of water diffusion and T1 values in peritumoural oedematous brain. Neuroreport 2002; 13 : 1335-40.

30 Phatak $P$, Shaldivin A, King LS, Shapiro $P$, Regenold WT. Lithium and inositol: effects on brain water homeostasis in the rat. Psychopharmacology (Berl) 2006; 186: 41-7.

31 Bearden CE, Thompson PM, Dalwani M, Hayashi KM, Lee AD, Nicoletti M, et al. Greater cortical gray matter density in lithium-treated patients with bipolar disorder. Biol Psychiatry 2007; 62: 7-16.

32 Bowley MP, Drevets WC, Ongür D, Price JL. Low glial numbers in the amygdala in major depressive disorder. Biol Psychiatry 2002; 52: 404-12.

33 Frazier JA, Breeze JL, Papadimitriou G, Kennedy DN, Hodge SM, Moore CM, et al. White matter abnormalities in children with and at risk for bipolar disorder. Bipolar Disord 2007; 9: 799-809.

34 Adler CM, Adams J, DelBello MP, Holland SK, Schmithorst V, Levine A, et al. Evidence of white matter pathology in bipolar disorder adolescents experiencing their first episode of mania: a diffusion tensor imaging study. Am J Psychiatry 2006; 163: 322-4.

35 Waxman EA, Lynch DR. N-methyl-D-aspartate receptor subtypes: multiple roles in excitotoxicity and neurological disease. Neuroscientist 2005; 11: $37-49$.

36 McDonald JW, Levine JM, Qu Y. Multiple classes of the oligodendrocyte lineage are highly vulnerable to excitotoxicity. Neuroreport 1998; 9: 2757-62.

37 McEwen B. Glucocorticoids, depression, and mood disorders: structural remodeling in the brain. Metabolism 2005; 54: 20-3.

38 Van der Schot AC, Vonk R, Brans RG, van Haren NE, Koolschijn PC, Nuboer V, et al. Influence of genes and environment on brain volumes in twin pairs concordant and discordant for bipolar disorder. Arch Gen Psychiatry 2009 66: $142-51$. 